\title{
Enucleation Induces Parvalbumin and Glial Fibrillary Acidic Protein, but Not Calbindin D28k Protein Expression in Superior Colliculus of Wistar Rats
}

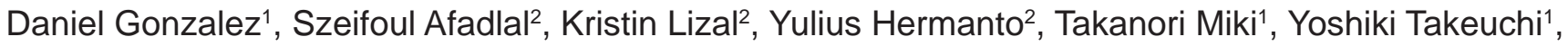 \\ Irawan Satriotomo ${ }^{1,2}$ \\ ${ }^{1}$ Department of Anatomy and Neurobiology, Faculty of Medicine, Kagawa University, Kagawa, Japan \\ ${ }^{2}$ Indonesia Brain Research Center (IBRC), Surya University, Tangerang, Indonesia
}

Background: It is known that eye enucleation causes various morphological and functional alterations in the central nervous system (CNS). The purpose of this study was to examine the sub-chronic effects of monocular enucleation on the distribution of the calcium binding proteins calbindin D28k (CB) and parvalbumin (PV) as well as the glial fibrillary acidic protein (GFAP) immunoreactivity in the superior colliculus (SC) of Wistar rats.

Materials and Methods: Thirty young adult (8 weeks) male Wistar rats from SLC (Shizuoka, Japan), weighing 200-250 grams, were housed in separate cages under controlled conditions with a constant temperature kept in 12:12 light/dark cycle and ad libitum water and food. In this study the rats were divided into two groups, a control and an enucleated groups. The experimental group received unilateral eye enucleation and was allowed 1, 4 or 12 weeks recovery before sacrificed.

Results: Unilateral enucleation over a period of 1 week or more caused a decrease in the number CB-immunoreactive (CBIR) neurons. This loss was associated with an increase in GFAP-IR astrocytes in the superficial gray layer and the optic layer of the SC with contralateral side predominance. In addition, the CB-IR neurons illustrated a smaller soma and poor dendritic arborization. Conversely, the GFAP-IR astrocytes were hypertrophied with longer foot processes on the contralateral side of enucleation. Interestingly, the number of PV-IR neurons was elevated for up to 4 weeks in enucleated rats versus shamoperated rats.

Conclusion: This study demonstrates the importance of calcium-binding protein homeostasis and reversible glial response for maintaining variability of neuronal function in sub-cortical visual centers following optic nerve deafferentation.

Keywords: enucleation, superior colliculus, calbindin D28k, parvalbumin, glial fibrillary acidic protein

\section{Introduction}

Enucleation is a well-established model for the study of Wallerian or transneuronal degeneration and produces significant structural and physiological change in the central nervous system (CNS). ${ }^{1,2}$ Studies of cell number following ablation in CNS or of peripheral sense organs have established that the availability of both synaptic targets and synaptic input can be important for cell survival. For instance, it has been shown that a decrease in competition of the target cells in the CNS increases cell survival in remaining populations. ${ }^{1}$ In this model we focus on the sub-

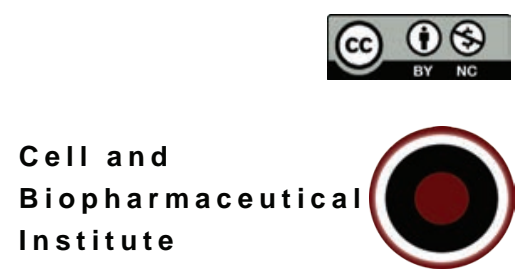

Cell and Institute 
cortical level of visual input, which travels to the cortex by either the retino-geniculate pathway or via the retinocollicular pathway. The retino-collicular route terminates in the superior colliculus (SC) where the processing of visual and other sensory information results in the generation of the orientation response towards novel sensory stimuli. ${ }^{2}$

Calcium binding proteins have been implicated as important regulators of neuronal degeneration in pathological processes. ${ }^{3}$ Calbindin D28k (CB), a member of the super family of calcium binding proteins is implicated in the regulation of intracellular calcium and can be a marker of several neuronal subpopulations. ${ }^{4}$ In the adult mammalian brain, CB is believed to serve a neuroprotective role by its calcium-buffering abilities. ${ }^{5}$ Recent evidence suggests that neurons containing the calcium binding proteins $\mathrm{CB}$ and parvalbumin (PV) have different distributions, matching respectively the distribution of $\mathrm{W}$ and $\mathrm{Y}$ retinal ganglion cell inputs to the cat SC. ${ }^{6}$ We hypothesize that monocular enucleation will affect calcium buffering in the SC neurons, altering mechanisms of $\mathrm{CB}$ and $\mathrm{PV}$ adaptation following neuronal injury.

In addition to neuronal adaptation after injury, significant astrocyte adaptation occurs. There have been many recent reports concerning the functions of astrocytes following injury, including neurotransmitter uptake as well as synthesis and secretion of neurotrophic factors. ${ }^{7,8}$ Astrocytes responding to an insult of the CNS enter a state of enhanced activation, becoming "reactive astrocytes." Glial fibrillary acidic protein (GFAP), a major intermediate filament protein of astrocytes is an antigen commonly used to examine the distribution of astrocytes. GFAP levels are a reliable indicator of the onset and progression of reactive astrocytes in response to neuronal degeneration or injury. ${ }^{9}$ The present report extends descriptions of the alterations of neurons and astrocytes, as well as quantitative changes of CB-immunoreactive (CB-IR), PV-IR neurons and GFAP-IR astrocytes in this neurodegenerative injury model.

\section{Materials and methods}

\section{Immunohistochemistry procedures}

Thirty young adult (8 weeks) male Wistar rats from SLC (Shizuoka, Japan), weighing 200-250 grams, were housed in separate cages under controlled conditions with a constant temperature $\left(23 \pm 1^{\circ} \mathrm{C}\right)$, kept in 12:12 light/dark cycle and ad libitum water and food. In this study the rats were divided into two groups, a control $(n=8)$ and an enucleated groups. The experimental group $(n=24)$ received unilateral eye enucleation and was allowed 1,4 or 12 weeks ( $\mathrm{n}=8$ for each groups) recovery before sacrificed. As previously described, ${ }^{10,11}$ treated rats underwent monocular eye enucleation by dissection of periorbital tissue with the Irish scissors, resecting the optic nerves, removing the left eye and suturing the eye lid to prevent the bleeding. All surgeries were conducted under deep anesthesia with sodium pentobarbital $(60 \mathrm{mg} / \mathrm{kg})$ injected intraperitoneally. The experimental procedures were conducted in accordance with the National Institute of Health (NIH) Guide for Care and Use of Laboratory Animals (NIH Publication No. 8023, revised 1996). The approval of the Kagawa Medical University Animal Committee was obtained for this study and every effort was made to minimize both animal number and suffering.

For tissue collection, animals were anaesthetized with sodium pentobarbital (40mg/kg i.p.) then perfused intracardially with $0.02 \mathrm{M}$ phosphate buffered saline (PBS) followed by a fixative of $4 \%$ paraformaldehyde in PBS. Brains were removed and cryoprotected in $30 \%(\mathrm{w} / \mathrm{v})$ sucrose at $4^{\circ} \mathrm{C}$ until it sank. Serial $40 \mu \mathrm{m}$-thick frozen sections were cut coronally and processed for immunohistochemistry. Briefly, to stain for either CB, PV or GFAP, free floating sections were incubated in the following: $10 \%$ normal goat serum in PBS with $0.2 \%$ Triton X-100 (0.02 M PBS-T, pH 7.4) for 60 min, anti-CB mouse monoclonal antibody (1:500 dilution, Sigma chemical company, St. Louis, Missouri, USA), or anti-PV mouse monoclonal antibody (1:200 dilution, Sigma chemical company, St. Louis, Missouri, USA) or anti-GFAP rabbit polyclonal antibody (1:2000 dilution, DAKO, Glostrup, Denmark) overnight at $4^{\circ} \mathrm{C}$. As a second antibody biotinylated anti-mouse IgG for CB and $\mathrm{PV}$, or biotinylated anti-rabbit IgG for GFAP in PBS were used for $60 \mathrm{~min}$ at room temperature (RT). After further rinse with PBS, the secondary antibodies were localized by the avidin-biotin complex method (Vectastain, Vector Lab. Burlingame, California, USA) for 60 min at RT. The location of the antibody-bound peroxidase was then visualized by incubation with the substrate 3,3-diaminobenzidine (DAB) (Dosindo, Osaka, Japan) peroxidase under visual control. Stained sections were mounted on gelatin-coated glass slides and air-dried. Tissue were then dehydrated, cleared and cover-slipped using Entellan (Merck, Darmstadt, Germany). Control sections omitting the primary and secondary antibodies were processed identically and consistently yielded no labeling. The morphology and 
expression of CB, PV and GFAP were studied with the aid of a light microscope (Nikon, YTHM, Tokyo, Japan).

\section{Quantitative procedures}

For a morphometric study of CB-IR, PV-IR neurons and GFAP-IR astrocytes were counted in superficial gray layer (SGL) and optic layer (OL) of superficial layer (SL) of SC, based upon a rat brain atlas from plate 40 (Bregma -5.60) until plate 46 (Bregma -7.64). ${ }^{12}$ The counting frame (350 $\mu \mathrm{m}$ x $240 \mu \mathrm{m}$ ) was set in the middle part of both layers (Figure 1B). The labeled cells were counted semi-quantitatively in sections of each group. An initial section that contained any sign of the SC was numbered as the first section, with all subsequent sections being numbered sequentially. For each animal, a number between 1 and 4 was selected randomly in order to determine first section. Every $4^{\text {th }}$ sections were picked up and the number of CB-IR neurons $\left(1^{\text {st }}, 5^{\text {th }}, 9^{\text {th }}\right.$ etc. $)$, PV-IR neurons $\left(2^{\text {nd }}, 6^{\text {th, }} 10^{\text {th }}\right.$ etc. $)$ and GFAP-IR astrocytes $\left(3^{\text {rd }}, 7^{\text {th }}, 11^{\text {th }}\right.$ etc. $)$ were counted.

Approximately 10 sections from each animal $(n=8)$ per groups were used in this study. The photographs of $C B$, PV and GFAP ( $x 40$ objective lens) that taken from selected sections with light microscope were captured by chargecoupled device (CCD) color camera, digitized and uploaded into a personal computer with the aid of image analysis software. Photographs of individual cells were used in order to avoid interference from overlapping images and IR-positive cells within the counting frame were counted manually. The number of immunoreactive cells $/ \mathrm{mm}^{2}$ tissue from both layer of contralateral SC were calculated and then compared with the respective ipsilateral side.

\section{Statistical analysis}

The statistical analysis of data from each side was performed by two-way analysis of variance (ANOVA). Post hoc tests were carried out where appropriate using Tukey-Kramer's test. For two groups, the comparison was made by Student's T-test for paired and unpaired observation as appropriate. All statistical analyses were performed using Minitab software (Abacus Concepts, California, USA) with $p$ value being less than 0.05 was considered significant.

\section{Results}

In mammals the SC has seven layers, which on functional and anatomical grounds can be considered two interacting tiers. The three most-dorsal layers (zonal layer (ZL), SGL and OL are known collectively as the SL (Figure 1A). SL neurons receive inputs from contralateral retinal ganglion cells and are organized retinotopically. ${ }^{2}$ The others, four deep layers (intermediate gray layer (IGL), intermediate white layer (IWL), deep gray layer (DGL) and deep white layer (DWL)) receive little direct visual input, ${ }^{13,14}$ but are also organized retinotopically. Because the SL of SC receives direct visual input, we focused on the SL in this manuscript.

\section{CB immunoreactivity}

In the sham-operated or control rat, intense CB-IR was observed throughout the dorsal layer (Figure 1A, Figure 1B). Small, round neurones, widely dispersed in the SGL (small panel), were the most distinctive feature in CB-IR sections. However, large and densely packed multipolar neurones in the OL also exhibited CB-IR in their fine processes (small panel). The number of CB-IR neurons in SGL and OL varies slightly, with more found in the SGL (Table 1).

At 1-week post-enucleation, the number of CB-IR neurons in the ipsilateral SC was not significantly different from sham-operated rats (Figure 1C). However, in the contralateral SGL, CB-IR was observed in neuronal somas, but not processes (Figure 1D; small panel). Moreover, the number of CB-IR neurons is decreased significantly, when compared to sham-operated rats, on the contralateral side in both SGL and OL (330.312.4 vs. 427.3 \pm 20.7 and 339.8 \pm 19.4 vs. $401.4 \pm 32.5, p<0.05$; respectively).

At 4-weeks post-enucleation, a marked reduction of CB-IR neurons was present on both sides with contralateral predominance (Figure 1E, Figure 1F; small panel). Quantitatively, their number were down to $60 \%$ in the SGL $(262.6 \pm 20.4$ vs. $427.3 \pm 20.7, p<0.05)$ and $65 \%$ in the OL $(259.5 \pm 18.9$ vs. $401.4 \pm 32.5, p<0.05)$ compared to control or sham groups. The number of CB-IR neurons on the ipsilateral side also decreased to $74 \%$ in the SGL (318.4 \pm 18.2 vs. $429.4 \pm 19.2, p<0.05)$ and $73 \%$ in the OL $(292.7 \pm 22.5$ vs. $400.0 \pm 35.9, p<0.05)$. As seen in Figures $1 \mathrm{E}$ and Figure $1 \mathrm{~J}$, the number of CB-IR neurons on the contralateral side of both SGL and OL were decreased 1 or 4 weeks after enucleation, but do not further decrease 4-12 weeks post-enucleation.

At 12 weeks post-enucleation (Figure 1G, Figure 1H), the SC on the contralateral side appeared slightly atrophic (double arrow head) and the number of CB-IR neurons continuously decreased compared to control animals. At higher magnification, the immunodensity of CB-IR neurons 


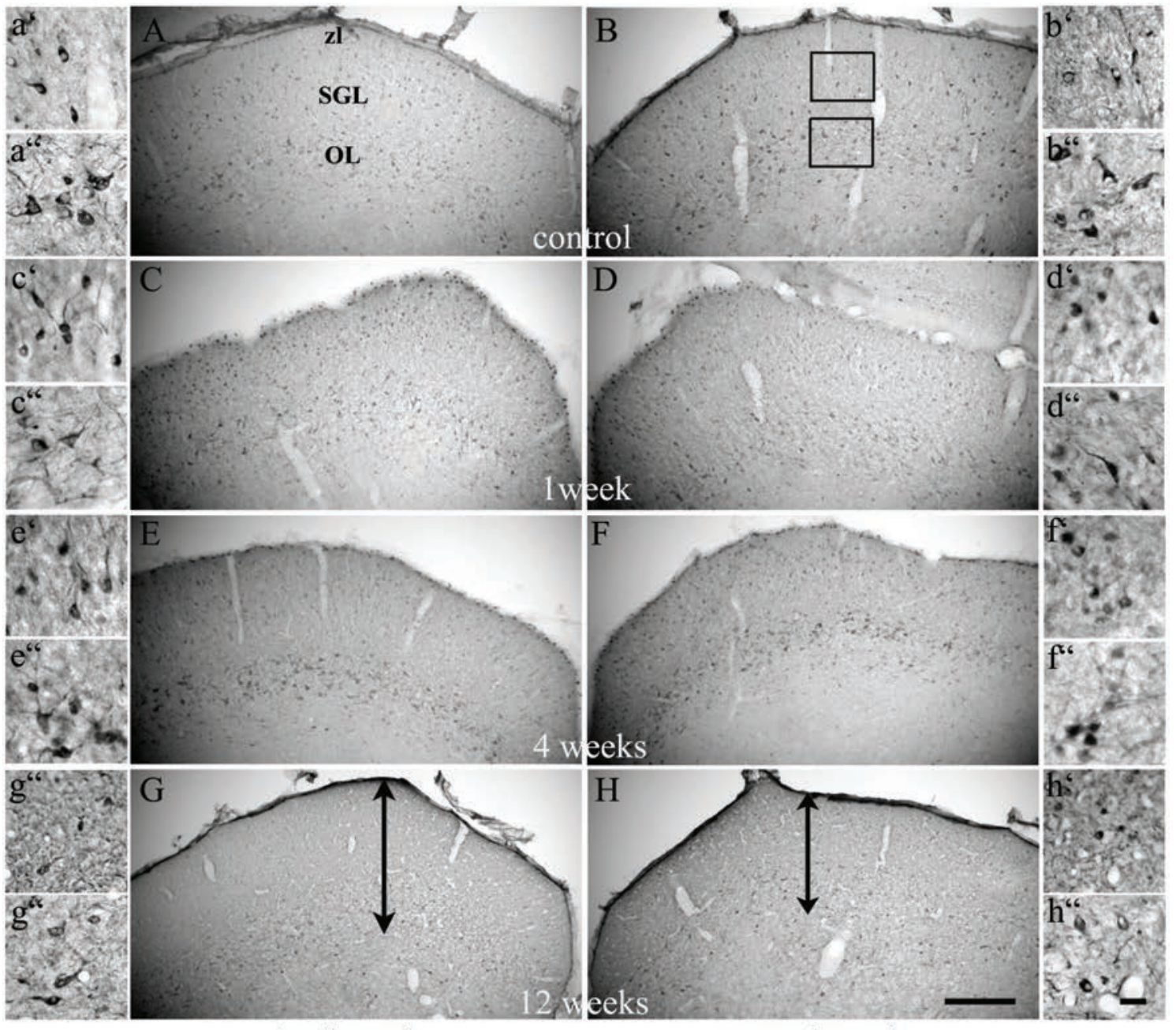

ipsilateral

\section{I}

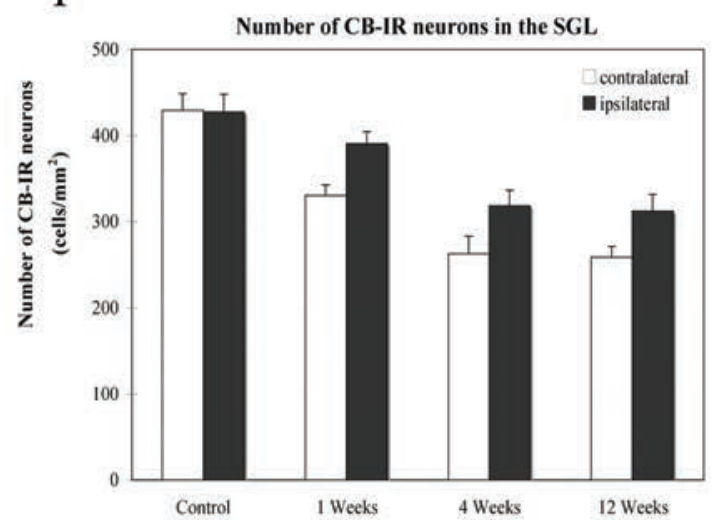

contralateral

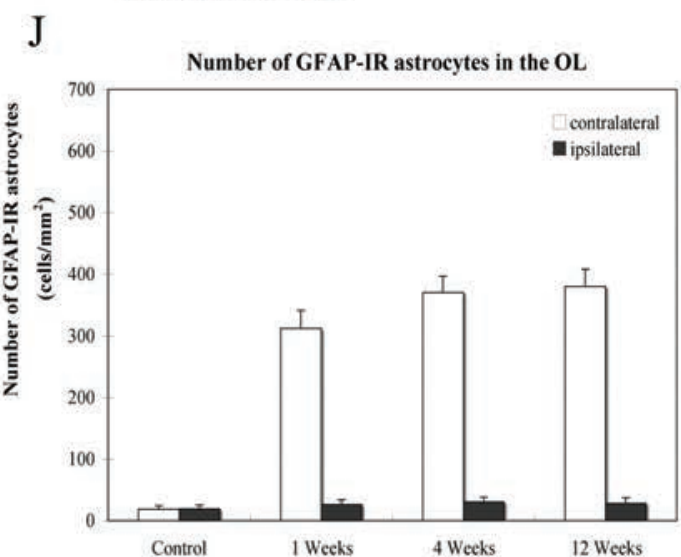

Figure 1. Photomicrograph of CB-IR neurons in the SC of control (A, B) and enucleated rats at 1, 4, 12 weeks after unilateral enucleation, respectively (C-H). Small panel are representative of magnified field of CB-IR neurons in SGL (upper) and OL (lower) of SC in each time points. The rectangle areas show frame set on SGL and OL for counting the cells number (B). Note, the CB-positive neurons have intense labelling in the SGL and OL in sham-operated rats. In enucleated rats, The CB expression is reduced and their number decreased time dependently with contralateral predominance (D, F, H) compared to the ipsilateral side (C, E, G). Graphic of morphometric analysis showed the CB-IR neuronal density in SGL (I) and OL (J) is significantly decreased after optic nerve deafferentiation compared to sham-operated rat. Scale bar: $200 \mu \mathrm{m} ; 50 \mu \mathrm{m}$ in small panel. 
on the contralateral side of both SGL and OL demonstrated a weak labelling in cell bodies and processes (small panel). Statistical analysis of numerical density of CB-IR neurons in SGL and OL showed significant main effects over time, side and time x side interaction (Table 1, Table 2).

\section{PV immunoreactivity}

PV-IR neurones in the SGL of control rats are small and widely scattered, with weak staining (Figure 2A, Figure 2B). In many instances their cell processes were difficult to visualise. On the other hand, some of PV-IR neurones in the $\mathrm{OL}$ are more immunoreactive. At higher magnification, these neurons were frequently bipolar in shape and oriented parallel to the piamater of the SC (small panel).

At 1-week post-enucleation, the PV positive neurons showed stronger homogenous PV labelling (Figure 2C, Figure 2D) and their number significantly increased in both SGL and OL with contralateral side predominance (459.5 \pm 28.2 vs. $191.7 \pm 17.2$ and $147.7 \pm 16.6$ vs. $114.3 \pm 10.0$, $p<0.05$, respectively). At higher magnification, the cell bodies and processes appeared to be stained intensely when compared to the ipsilateral side (small panel).

At 4 weeks post-enucleation, the number of PVpositive neurons was continuously increased in the contralateral side of SGL $(531.7 \pm 34.4$ vs. $191.7 \pm 17.2$, $p<0.05)$ and in the OL $(143.1 \pm 13.8$ vs. $114.3 \pm 10.0, p<0.05)$, compared to the control groups (Figure 2E, Figure 2F). These PV-IR neurons also exhibited more immunoreactivity in their staining (small panel). Moreover, PV-IR neurons on the ipsilateral side of SGL also begin showing to increase (260.2 \pm 17.1 vs.190.8 $\pm 13.9, p<0.05)$.

At 12 weeks after enucleation, the PV immunoreactivity was still prominent compare to the control group (Figure 2G, Figure 2H). As shown in Figure 2I and Figure 2J, the number of PV-IR neurons on the contralateral SGL was increased up to 4 weeks before decline at 12 weeks. The up-regulation of PV-IR in the OL has a shorter time course, with the PV-immunopositive neurons increasing only up to 1-week post-enucleation before its decline. Quantitative analysis of PV-IR neurons in SGL and OL confirmed the significant main effects over time, side and time $\mathrm{x}$ side interactions (Table 1, Table 2).

\section{GFAP immunoreactivity}

In control rats, GFAP-positive astrocytes were observed mostly in the ZL of SL of the SC. Morphologic characteristics of these astrocytes suggested that they were mainly of the protoplasmic type. They were smaller than those in enucleated rats, with fewer and shorter processes (Figure 3A, Figure 3B). In the SGL and OL, GFAP labelled astrocytes were few and located only around the capillary (small panel). After unilateral enucleation, an increase in GFAP-IR astrocytes was found bilaterally in the SL of SC, but more prominently on the contralateral side. Notable changes at each survival time are described below.

At 1-week post-enucleation, astrocytes reaction on the ipsilateral side was mostly limited to the ZL nearing the optic tract (Figure 3C). Reactive astrocytes were evident in the SGL only on the contralateral side (Figure 3D). Astrocytes number significantly increased in both SGL and OL with contralateral side predominance $(501.4 \pm 42.9$ vs. $23.1 \pm 6.5$ and $312.0 \pm 28.1$ vs. 18.8 $\pm 6.3, p<0.05$; respectively). These astroglial responses in the SGL consisted of thick, coarse and heavily stained bundles and extended to the middle layer of the SC. Under higher magnification, this injury response appeared as significant astrocytic hypertrophy and proliferation (small panel).

At 4 weeks post-enucleation, hypertrophy of astrocytes appeared less obvious when compared to 1 week (Figure 3E, Figure 3F), whereas the numbers of GFAP-IR astrocytes further increased (Figure 3I, Figure 3J). These labelled astrocytes in the SGL and OL have smaller somata but their processes remain longer (small panel).

The astrocytic reaction remained significant in all zones of the SL of SC at 12 weeks post-enucleation (Figure 3G, Figure 3H). The sizes of astrocytes somata were further reduced, although the number of GFAPpositive astrocytes was remain higher compared to the control rats (small panel). Surprisingly, the number of GFAP-positive astrocytes in SGL at 12 weeks (Figure 3I, Figure 3J) was reduced when compared to 4 weeks postenucleation ( $264.9 \pm 31.1$ vs. 553.2 $\pm 36.8, p<0.05)$. However, the number of GFAP-IR astrocytes in OL did increase but not significantly difference. A decrease in the sizes of the contralateral SC that may associate with the degenerated of the optic tract was also observed. Statistical analysis of the numerical density of GFAP-IR astrocytes in SGL and OL showed significant main effects with time, side and time $\mathrm{x}$ side interactions (Table 1, Table 2).

\section{Discussion}

Our experiments have shown that the unilateral eye enucleation alters the numbers of CB-IR neurons, PV-IR 


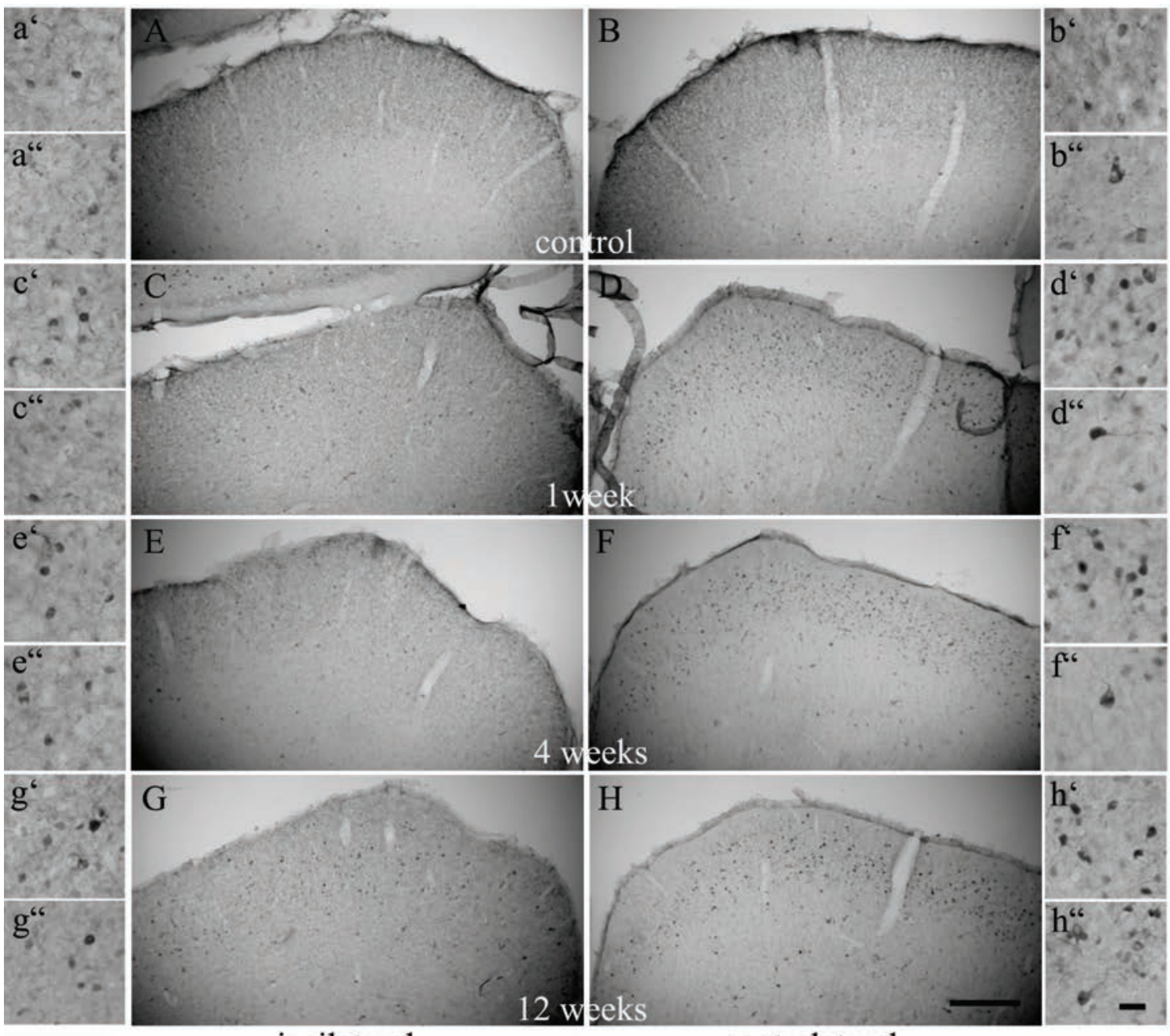

ipsilateral

contralateral
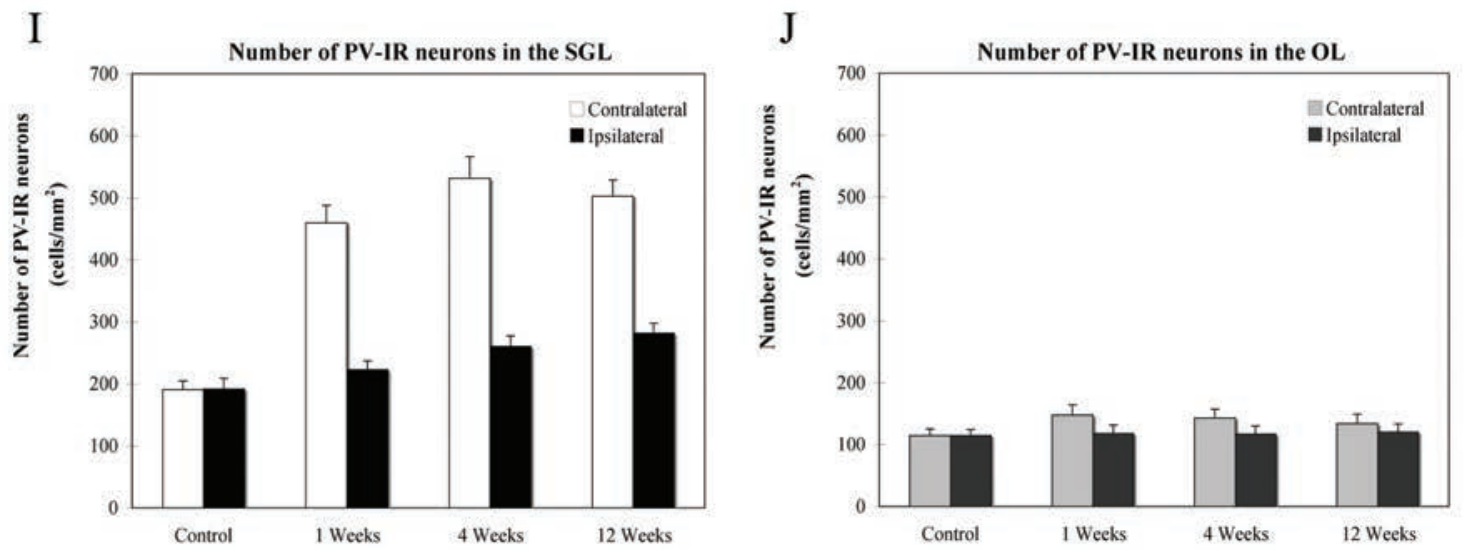

Figure 2. Photomicrograph of PV-IR neurons in the SC of control (A, B) and enucleated rats at 1, 4, 12 weeks post-enucleation, respectively (C-H). Small panel are representative of magnified field of PV-IR neurons in SGL (upper) and OL (lower) of SC in each time points. The PV-IR neurons are very few and have light labelling in the SGL and OL in sham-operated rats. In enucleated rats, The PV-IR is highly expressed and their number increased significantly with contralateral predominance $(\mathrm{D}, \mathrm{F}, \mathrm{H})$. Graphic of morphometric analysis showed the PV-IR neuronal density in SGL (I) and OL (J) is increased up to 12 weeks following monocular enucleation. Scale bar: 200 $\mu \mathrm{m} ; 50 \mu \mathrm{m}$ in small panel. 

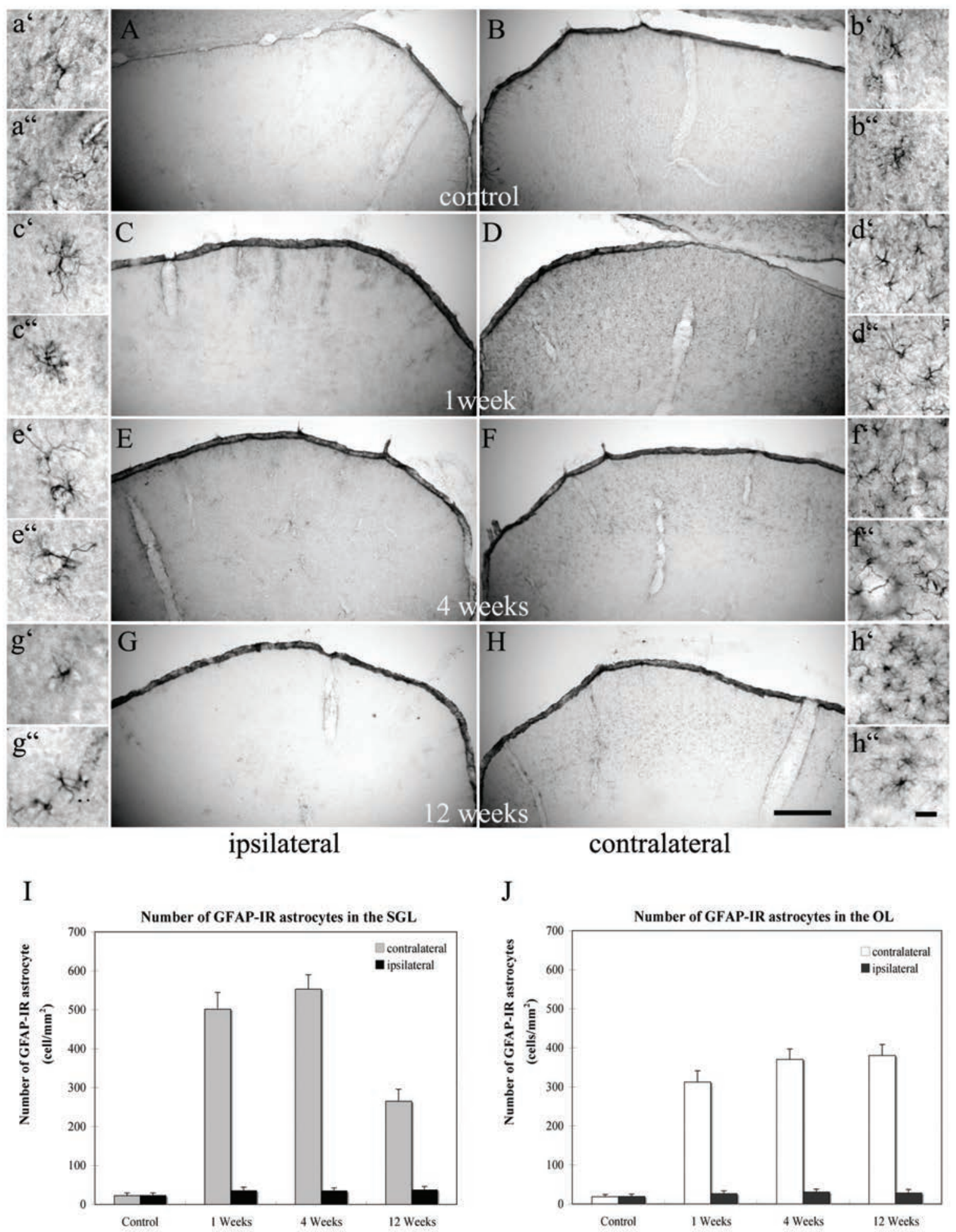

Figure 3. Photomicrograph of GFAP-IR astrocytes in the SC of control (A, B) and enucleated rats at 1, 4, 12 weeks post-enucleation, respectively (C-H). Small panel are representative of magnified field of GFAP-IR astrocytes in SGL (upper) and OL (lower) of SC in each time points. Note, the GFAP-IR astrocytes are very few and have small cell bodies and short processes in control rats (small panel). In enucleated rats, The GFAP-IR astrocytes are increased significantly in contralateral side (D, F, H) compared to the ipsilateral side (C, E, G). These reactive astrocytes have bigger cell bodies with longer processes (small panel). Graphic I and J represent morphometric analysis of GFAP-IR neuronal density in SGL and OL, respectively. Scale bar: $200 \mu \mathrm{m} ; 50 \mu \mathrm{m}$ in small panel. 
Table 1. Quantitative analysis of CB-IR, PV-IR, and GFAF-IR in SGL and OL. Data on the number of labeled-cells (cells/mm2) in the rat superior colliculus following monocular.

\begin{tabular}{|c|c|c|c|c|c|c|c|}
\hline \multirow{2}{*}{ Time } & \multirow{2}{*}{ Slide } & \multicolumn{2}{|c|}{ CB-IR } & \multicolumn{2}{|c|}{ PV-IR } & \multicolumn{2}{|c|}{ GFAF-IR } \\
\hline & & SGL & OL & SGL & OL & SGL & OL \\
\hline \multirow[t]{2}{*}{ Control $(n=8)$} & $\mathrm{R}$ & $429.4 \pm 19.2$ & $400.0 \pm 35.9$ & $190.8 \pm 13.9$ & $114.6 \pm 10.8$ & $22.6 \pm 6.8$ & $18.6 \pm 5.8$ \\
\hline & $\mathrm{L}$ & $427.3 \pm 20.7$ & $401.4 \pm 32.5$ & $191.7 \pm 17.2$ & $114.3 \pm 10.0$ & $23.1 \pm 6.5$ & $18.8 \pm 6.3$ \\
\hline \multirow[t]{2}{*}{1 week $(n=8)$} & I & $390.6 \pm 13.9$ & $396.2 \pm 18.6$ & $222.8 \pm 14.3$ & $117.8 \pm 13.5$ & $35.5 \pm 8.6$ & $26.3 \pm 7.3$ \\
\hline & $\mathrm{C}$ & $330.3 \pm 12.4$ & $339.8 \pm 19.4$ & $459.5 \pm 28.2$ & $147.7 \pm 16.6$ & $501.4 \pm 42.9$ & $312.0 \pm 28.1$ \\
\hline \multirow[t]{2}{*}{4 week $(n=8)$} & I & $318.4 \pm 18.2$ & $292.7 \pm 22.5$ & $260.2 \pm 17.1$ & $116.9 \pm 13.1$ & $34.8 \pm 7.7$ & $30.4 \pm 7.8$ \\
\hline & C & $262.6 \pm 20.4$ & $259.5 \pm 18.9$ & $531.7 \pm 34.4$ & $143.1 \pm 13.8$ & $553.2 \pm 36.8$ & $370.4 \pm 26.8$ \\
\hline \multirow[t]{2}{*}{12 week $(n=8)$} & I & $312.2 \pm 19.5$ & $286.8 \pm 19.5$ & $281.6 \pm 16.3$ & $120.0 \pm 13.2$ & $37.4 \pm 8.7$ & $28.2 \pm 8.9$ \\
\hline & $\mathrm{C}$ & $258.8 \pm 12.6$ & $254.3 \pm 14.9$ & $502.8 \pm 25.9$ & $133.8 \pm 14.9$ & $264.9 \pm 31.1$ & $380.2 \pm 27.9$ \\
\hline
\end{tabular}

Table 1. Results of two-way ANOVA of quantitative analysis of CB-IR, PV-IR, and GFAF-IR in SGL and OL.

\begin{tabular}{|c|c|c|c|c|}
\hline & \multicolumn{2}{|c|}{ SGL } & \multicolumn{2}{|c|}{$\mathrm{OL}$} \\
\hline & d.f. & F & d.f. & F \\
\hline \multicolumn{5}{|l|}{ (A) $\mathrm{CB}$} \\
\hline Side & 1, 392 & $57.17 * *$ & 1, 392 & $32.00 * *$ \\
\hline Time & 3, 392 & $259.24 * *$ & 3, 392 & $151.23^{* *}$ \\
\hline Side $\mathrm{x}$ Time & 3, 392 & $17.83^{* *}$ & 3, 392 & $5.00 *$ \\
\hline \multicolumn{5}{|l|}{ (B) PV } \\
\hline Side & 1, 392 & $1,365.17 * *$ & 1, 392 & $24.65 *$ \\
\hline Time & 3, 392 & $376.64 * *$ & 3, 392 & $5.46 *$ \\
\hline Side $x$ Time & 3, 392 & $154.66^{* *}$ & 3, 392 & $3.54 *$ \\
\hline \multicolumn{5}{|l|}{ (C) GFAP } \\
\hline Side & 1, 392 & $3,288.06 * *$ & 1, 392 & $3,664.37 * *$ \\
\hline Time & 3, 392 & $552.40 * *$ & 3, 392 & $471.46^{* *}$ \\
\hline Side $x$ Time & 3, 392 & $507.55^{* *}$ & 3, 392 & $419.14^{* *}$ \\
\hline
\end{tabular}

neurons and GFAP-IR astrocytes in the SL of rat SC with contralateral predominance. This data is consistent with our previous findings showing that enucleation induced neuronal degeneration and decreased numbers CB-IR neurons. ${ }^{10} \mathrm{We}$ also observed an increase of PV-IR neurons and GFAP-IR astrocytes in the lateral geniculate nucleus (LGN) complex following unilateral enucleation. ${ }^{11}$ Because the major source of fibres entering the SGL of the SC is from the retinal ganglion of the contralateral eye, a decrease of CB-IR neurones following acute monocular enucleation is likely the result of anterograde transneuronal degeneration. By contrast, PV-IR was apparently enhanced in the SC neurons as a likely compensatory mechanism to help maintain their calcium buffering in the survival neurons. It could be suggested that a decrease of one calcium binding protein, $\mathrm{CB}$, would lead to increase synthesis of other calciumbuffering agents, such as PV. ${ }^{1}$
In agreement with the previous reports on several mammalian SC, ${ }^{15-18}$ CB-positive neurons were reduced and PV-IR neurones increased following unilateral enucleation. Furthermore, other calcium binding proteins like calretinin, have also been reported to increase in rat, ${ }^{19}$ mouse $^{20}$ and hamster. ${ }^{16}$ On the contrary, some investigators have shown that eye removal did not alter the total number of CB-IR neurons in the SCs. ${ }^{21-23}$ These discrepancies could be explained in part by differences in age (e.g., adult vs. fetal), species (e.g., rat vs. monkey or rabbit), location (SC vs. LGN complex) or time post-enucleation. The above data suggesting that both $\mathrm{PV}$ and calretinin expression is more plastic than CB on retinal input after monocular enucleation. These changes of expression of calcium-binding protein within the SC neurons may therefore reflect the protective functional need on different environmental changes. Thus, our present study proves the importance of calcium-binding 
protein homeostasis and reversible glial response for maintaining variability of neuronal function in sub-cortical visual centers following optic nerve deafferentation.

Brain inflammatory response is triggered by activation of astrocytes, along with other cells of glial lineage such as microglia, in response various types of CNS injury. Its outcome is determined by releasing inflammatory mediators including cytotoxic signals from gliosis process. However, these processes depend on many additional factors such as the intensity and duration of the injury/insult. Depending on specific circumstances, the inflammatory response in the brain can also promote neuroprotection and regeneration. The function of astrocytes in regulating cerebral blood flow and providing nutrition to the neurons, neurotransmitter synthesis and maintaining synaptic function becoming increasingly being recognized. A glial reaction may affect the activities of SC neurons, and it may influence compensation mechanism of lesion-induced visual disorders.

We demonstrated in this manuscript that the induction of astrocyte hypertrophy and proliferation after enucleation might depend on the nature of the injury and survival time. Our data show that a substantial increase of GFAP-IR occurs in the contralateral part of the rat SC on the first week up to 4 weeks in the SGL and is still increased up to 12 weeks postenucleation in the OL. The timing of changes in astrocyte immunoreactivity in the SC after enucleation suggests that the astrocytic response to optic nerve denervation is characterised by hypertrophy during the first month and proliferation thereafter. Early changes in the astrocyte might aid SC neurones in surviving the sudden loss of synaptic activation, as well as possible cytotoxicity of glutamate released from degenerating of SC neurones and nerve optic fibres. ${ }^{24,25}$ Improved early survival time may result from the increase in the number of GFAP-IR astrocytes. It suggests an increase in GFAP synthesis may be functionally related to trophic factor availability and regeneration or restoration of connectivity. ${ }^{26}$ After 4 weeks post-enucleation, we showed that the cell bodies of the GFAP-IR astrocytes become less obvious. However, their cell processes were longer compared to the resting astrocytes in un-enucleated rats. Those long and thin processes of the astrocytes oriented within the limited space between the neurones and fibre bundles may make efficient contact with the neurones to maintain the neuronal function. ${ }^{27}$ Subsequently, the later changes in the astrocytes may be aimed at rebuilding the local environment, in order to facilitate the plasticity in the SC neurones activities and pattern of local synaptic connections. ${ }^{28}$

\section{Conclusion}

In conclusion, we have revealed a significant decrease of $\mathrm{CB}$ in the SL of SC following monocular enucleation. This loss is likely compensated by an increase in PV with additional support from nearby astrocytes. We suggested that such compensatory mechanisms of calcium homeostasis in SC neurons might play an important role following neuronal loss, neurodegenerative disease or injury.

\section{References}

1. Finlay BL, Sengelaub DR, Berian CA. Control of Cell Number in Developing Visual System. I. Effects of Monocular Enucleation. Brain Res. 1986; 393(1): 1-10.

2. Binns KE. The Synaptic Pharmacology Underlying Sensory Processing in the Superior Colliculus. Prog Neurobiol. 1999; 59(2): 129-59.

3. Airaksinen MS, Eilers J, Garaschuk O, Thoenen H, Konnerth A, Meyer M. Ataxia and Altered Dendritic Calcium Signaling in Mice Carrying a Targeted Null Mutation of the Calbindin D28k Gene. Proc Natl Acad Sci. 1997; 94(4):1488-93.

4. Pfeiffer B, Norman AW, Hamprecht B. Immunocytochemical Characterization of Neuron-Rich Rat Brain Primary Cultures: Calbindin D28K as Marker of a Neuronal Subpopulation. Brain Res. 1989; 476(1): 120-8.

5. Mattson MP, Rychlik B, Chu C, Christakos S. Evidence for calciumreducing and excito-protective roles for the calcium-binding protein calbindin-D28k in cultured hippocampal neurons. Neuron. 1991; 6(1): 41-51.

6. Mize RR. Calbindin $28 \mathrm{kD}$ and Parvalbumin Immunoreactive Neurons Receive Different Patterns of Synaptic Input in the Cat Superior Colliculus. Brain Res. 1999; 843(1-2): 25-35.

7. Furukawa S, Furukawa Y, Satoyoshi E, Hayashi K. Synthesis and Secretion of Nerve Growth Factor by Mouse Astroglial Cells in Culture. Bichem Biophys Res Commun. 1986; 136(1): 57-63.

8. Kimelberg HK, Katz DM. High-affinity Uptake of Serotonin into Immunocytochemically Identified Astrocytes. Science. 1985; 228(4701): 889-91.

9. Agarwala S, Kalil RE. Axotomy-induced neuronal death and reactive astrogliosis in the lateral geniculate nucleus following a lesion of the visual cortex in the rat. J Comp Neurol. 1998; 392(2): 252-63.

10. Gonzalez D, Satriotomo I, Miki T, Lee KY, Yokoyama T, Touge T, et al. Effects of Monocular Enucleation on Calbindin-D 28k and C-Fos Expression in the Lateral Geniculate Nucleus in Rats. Okajimas Folia Anat Jpn. 2005; 82(1): 9-18.

11. Gonzalez D, Satriotomo I, Miki T, Lee KY, Yokoyama T, Touge T, et al. Changes of Parvalbumin Immunoreactive Neurons and GFAP Immunoreactive Astrocytes in the Rat Lateral Geniculate Nucleus Following Monocular Enucleation. Neurosci Lett. 2006; 395(2): 149-154.

12. Paxinos G, Watson C. The Rat Brain in Stereotaxic Coordinate. Academic Press; 1986. 
13. Berson D, McIlwain J. Retinal y-cell Activation of Deep Layer Cells in Superior Colliculus of the Cat. J Neurophysiol. 1982; 47(4): 70014.

14. Mize R. Patterns of Convergence and Divergence of Retinal and Cortical Synaptic Terminals in the Cat Superior Colliculus. Exp Brain Res. 1983; 51(1): 88-96.

15. Cork RJ, Baber SZ, Mize RR. Calbindin D28k- and Parvalbuminimmunoreactive Neurons Form Complementary Sublaminae in the Rat Superior Colliculus. J Comp Neurol. 1998; 394(2): 205-17.

16. Kang YS, Park WM, Lim JK, Kim SY, Jeon CJ. Changes of Calretinin, Calbindin D28K and Parvalbumin-Immunoreactive Neurons in the Superficial Layers of the Hamster Superior Colliculus Following Monocular Enucleation. Neurosci Lett. 2002; 330(1): 104-8.

17. Mize RR, Luo Q, Butler G, Jeon CJ, Nabors B. The Calcium Binding Proteins Parvalbumin and Calbindin-D 28K form Complementary Patterns in the Cat Superior Colliculus. J Comp Neurol. 1992; 320(2): 243-56.

18. Mize R, Luo Q, Tigges M. Monocular Enucleation Reduces Immunoreactivity to the Calcium-binding Protein Calbindin $28 \mathrm{kd}$ in the Rhesus Monkey Lateral Geniculate Nucleus. Vis Neurosci. 1992; 9(5): 5471-82.

19. Arai M, Arai R, Sasamoto K, Kani K, Maeda T, Deura S, et al. Appearance of Calretinin-Immunoreactive Neurons in the Upper Layers of the Rat Superior Colliculus After Eye Enucleation. Brain Res. 1993; 613(2): 341-6.

20. Gobersztejn F, Britto LRG. Calretinin in the Mouse Superior Colliculus Originates from Retinal Ganglion Cells. Braz J Med Biol Res. 1996; 29(11): 1507-11.
21. Mize RR, Luo Q. Visual Deprivation Fails to Reduce Calbindin 28kd or GABA Immunoreactivity in the Rhesus Monkey Superior Colliculus. Vis Neurosci. 1992; 9(2): 157-168.

22. Jeon CJ, Pyun JK, Yang HW. Calretinin and Calbindin D28K Immunoreactivity in the Superficial Layers of the Rabbit Superior Colliculus. Neuroreport. 1998; 9(17): 3847-52.

23. Lane RD, Allan DM, Bennett-Clarke CA, Rhoades RW. Differential Age-dependent Effects of Retinal Deafferentation upon Calbindinand Parvalbumin-immunoreactive Neurons in the Superficial Layers of the Rat's Superior Colliculus. Brain Res. 1996; 740(1-2): 208-14.

24. Sakurai T, Miyamoto T, Okada Y. Reduction Glutamate Content in Rat Superior Colliculus After Retino-tectal Denervation. Neurosci Lett. 1990; 109(3): 299-303.

25. Sakurai T, Okada Y. Selective Reduction of Glutamate in the Rat Superior Colliculus and Dorsal Lateral Geniculate Nucleus after Contralateral Enucleation. Brain Res. 1992; 573(2): 197-203.

26. Schwartz JP, Nishiyama N. Neurotrophic Factor Gene Expression in Astrocytes during Development and Following Injury. Brain Res Bull. 1994; 35(5-6): 403-7.

27. Hatten ME, Mason CA, Liem RKH, Edmondson JC, Bovolenta P, Shelanski ML. Neuron-astroglial Interactions in vitro and Their Implications for Repair of CNS Injury. Cent Nerv Syst Trauma. 1984; 1(1): 115-27.

28. Smith SA, Bedi KS. Unilateral Enucleation of Adult Rats does not affect the Synapse-to-Neuron Ratio within the Stratum Griseum Superficiale of the Superior Colliculus. Vision Res. 1998; 38(20): 3041-50. 